\title{
ILDAR ABDULLOVICH IBRAGIMOV
}

I. A. Ibragimov turned 80 on July 15, 2012. Ildar Abdullovich is a worldwide renowned mathematician, one of the leading specialists in Probability and Statistics.

I. A. Ibragimov is the author of four monographs; he heads a school of Probability and Statistics recognized in the world. Starting from his student years and till now he has been a leading researcher in this field.

I. A. Ibragimov introduced and studied in detail conditions of uniformly strong mixing; he proved the Central Limit Theorem for sums of weakly dependent random variables. In the spectral theory of stationary random processes, he indicated a connection between the property of total regularity of processes and the smoothness of the spectral density. In the domain of classical limit theorems, I. A. Ibragimov obtained necessary and sufficient conditions for power rate of convergence in the Central Limit Theorem for sums of i.i.d. random variables and necessary conditions under which the Chebyshev-Cramér asymptotic expansions are valid. He developed a new effective method of asymptotic analysis of functionals of random walks. His extremely fruitful collaboration with R. Z. Khasminskii lead to development of the asymptotic theory of statistical estimation for irregular families of distributions. The asymptotic model of a signal in white noise studied by I. A. Ibragimov and R. Z. Khasminskii became canonical in problems of nonparametric estimation and hypotheses testing. I. A. Ibragimov got deep results on estimation of analytic functions in statistical models and analytic spectral densities for Gaussian stationary processes. One of his new papers is published in this volume.

I. A. Ibragimov is a Full Member of Russian Academy of Sciences; he was awarded the Lenin prize and the Friendship order. After the death of his teacher, Yu. V. Linnik, I. A. Ibragimov became the permanent head of Laboratory of statistical methods at Leningrad-St. Petersburg Department of the Steklov Mathematical Institute; he was the Director of the Institute in 2000-2006. More than 50 years I. A. Ibragimov teaches at the Faculty of Mathematics and Mechanics of Leningrad-St. Petersburg University; in 1997-2005, he was the head of the Chair of Probability and Statistics.

In the hard years in the life of our country, I. A. Ibragimov managed not only to preserve the St. Petersburg scientific school of Probability and Statistics but also to fix its leading position in the Russian and world science. The influence of I. A. Ibragimov creates in scientific collectives which he heads a favorable research atmosphere and permanent high ethic standards. For the last 40 years, he heads the St. Petersburg seminar on Probability and Statistics, preserving friendly and creative atmosphere of scientific research.

Witnessing the work of I. A. Ibragimov, young researchers learn how to write high level papers and monographs and to give lectures and scientific talks. For decades, he is one of the best lecturers at the Faculty of Mathematics and Mechanics of St. Petersburg University.

I. A. Ibragimov is a person of unique erudition. He combines research in mathematics with deep interest in history of science; he is fascinated by literature, music, and poetry. He is attentive to sciences which seem distant from his basic speciality. Ildar Abdullovich loves life, he is optimistic and devoted to his profession. His judgments on actual life problems are always deep and independent. They are sometimes unexpected and even paradoxical, but always interesting and argumented.

The editors of this volume and the authors of the papers express their love, admiration, and deepest respect to Ildar Abdullovich; they wish him liveliness, strong health, and new scientific successes. Many of us, including the pupils of I. A. of different generations, started our scientific life and realized themselves in this life due to I. A. Ibragimov. We are forever grateful to him for that.

Translated by S. Yu. Pilyugin. 